Tropical Journal of Pharmaceutical Research June 2010; 9 (3): 243-249

(C) Pharmacotherapy Group,

Faculty of Pharmacy, University of Benin

Benin City, 300001 Nigeria.

All rights reserved.

Research Article

Available online at http://www.tjpr.org

\title{
Synergy between Phenothiazines and Oxacillin against Clinical Isolates of Methicillin-Resistant Staphylococcus aureus
}

\author{
Sanaz Hadji-nejad ${ }^{1}$, Mohammad Rahbar ${ }^{2}$ and Hadi Mehrgan ${ }^{1 *}$ \\ ${ }^{1}$ Department of Pharmaceutics, School of Pharmacy and Pharmaceutical Sciences Research Center, Shahid \\ Beheshti University of Medical Sciences, ${ }^{2}$ Health Reference Laboratories, Ministry of Health, Tehran, Iran.
}

\begin{abstract}
Purpose: To evaluate the antimicrobial and resistance-reversal activities of seven phenothiazine derivatives against one standard methicillin-sensitive and ten methicillin-resistant Staphylococcus aureus (MRSA) strains originating from human infections.

Methods: Minimum inhibitory concentrations (MIC) of the compounds were determined by agar dilution method, and synergy between phenothiazines and oxacillin was investigated using Checkerboard (microbroth dilution) technique.

Results: We found that all $S$. aureus strains, regardless of their susceptibility to oxacillin, were inhibited by phenothiazines at a concentration of $8-256 \mu \mathrm{g} / \mathrm{mL}$, with thioridazine being the most potent inhibitory agent. Phenothiazines at sub-inhibitory concentrations lowered the MIC of oxacillin from 256 to $2 \mu \mathrm{g} / \mathrm{mL}$, which is a clinically significant level. The highest number of synergistic combinations, i.e., fractional inhibitory concentration (FIC) index less than 0.5 , was seen with chlorpromazine and perphenazine. However, thioridazine reversed antibiotic resistance at a concentration as low as $4 \mu \mathrm{g} / \mathrm{mL}$.

Conclusion: Although synergy was observed at concentrations higher than those that phenothiazines usually attain in vivo, the potential offered by non-antibiotics justifies further animal experiments as well as clinical trials to establish their clinical relevance.
\end{abstract}

Keywords: Methicillin-resistance, Staphylococcus aureus, Oxacillin; Phenothiazines, Non-antibiotics, Synergy. 


\section{INTRODUCTION}

Antibiotics and antimicrobial chemotherapeutics exist in large numbers in today's pharmaceutical market. This notwithstanding, their use is becoming increasingly restricted due largely to the development of drug resistance among microorganisms coupled with the toxicity of several antimicrobials. This has necessitated the search for newer antimicrobial agents that would overcome these drawbacks.

Several compounds belonging to various pharmacological classes possess moderate to powerful antibacterial activity. Such compounds, which possess antimicrobial properties in addition to their predesignated pharmacological actions, have been called the 'Non-antibiotics' [1]. The antimicrobial activity of synthetic and nonchemotherapeutic compounds such as methylene blue has been known since the days of Ehrlich (1854 - 1915). Phenothiazines are dopamine receptor agonists which are used clinically as antihistaminic agents or neuroleptics for the management of psychosis and have been shown to have modest antimicrobial activities against a wide array of microorganisms when combined at one-fourth of their MICs [2]. With common antimicrobial substrates of multi-drug resistance (MDR) pumps of Staphylococcus aureus, phenothiazines augmented the antimicrobial activities of these substrates [3]. In this regard, phenothiazines could be used as adjuncts to antibiotics where resistance has been observed [2].

In this study, the antimicrobial effects of seven phenothiazines and their possible synergy with oxacillin against clinical MRSA isolates were investigated.

\section{EXPERIMENTAL}

\section{Bacteria}

The 10 methicillin-resistant Staphylococcus aureus (MRSA) strains used in this study were obtained from routine specimens at Milad Hospital of Tehran, Iran, and were designated as $M_{1}-M_{10}$. In addition, methicillin-sensitive $S$. aureus (MSSA) ATCC $29213\left(\mathrm{mecA}^{-}\right)$and MRSA ATCC 33591 (mec $\mathrm{A}^{+}$) were procured form Mast Group Ltd, Merseyside, UK and used as standard strains. All bacteria were preserved both in deep frozen state and at $4{ }^{\circ} \mathrm{C}$ in slant agar.

\section{Materials}

Oxacillin (as sodium salt) was purchased from Sigma, St. Louis, USA. The phenothiazines used - chlorpromazine, trifluoperazine $\mathrm{HCl}$, thioridazine $\mathrm{HCl}$, perphenazine, fluphenazine $\mathrm{HCl}$, promethazine and thiethylperazine maleate - manufactured by Aventis Pharma, Industrial Chimica Milanesa, Trifarma, Industrial Chimica Milanesa, Fine Chemicals Corporation, Egis Pharmaceuticals Ltd and Novartis, respectively. These drugs were provided free of charge by their local subsidiaries in Iran. All the agents were stored at $4{ }^{\circ} \mathrm{C}$ over silica gel and used as received. Mueller Hinton agar (MHA), Mueller-Hinton broth (MHB) and trypticase soy agar (TSA) media were obtained from Merck Co, Germany. All other materials were of analytical grade.

\section{Determination of the minimum inhibitory concentration (MIC) of the drugs}

The agar dilution method recommended by the Clinical and Laboratory Standards Institute (CLSI) was employed to determine the MIC of antimicrobial agents [4]. Oxacillin was dissolved in sterile distilled water, filtersterilized and then added to molten MHA containing $2 \% \mathrm{NaCl}$ at concentrations of 0.5 to $1024 \mu \mathrm{g} / \mathrm{mL}$. It was then poured into sterile Petri dishes under aseptic conditions and maintained at $\mathrm{pH} 7.2$ - 7.4. The phenothiazines, except perphe-nazine and thiethylperazine, were also dissolved in sterile distilled water and filter-sterilized. Perphenazine was dissolved in dimethyl sulfoxide (DMSO) and thiethylperazine in methanol together with a few drops of 
ammonia. The phenothiazine solutions were finally added to molten MHA to make final concentrations of 2 to $1024 \mu \mathrm{g} / \mathrm{mL}$, and treated as described above for oxacillin.

Bacterial strains were grown in TSA at $35 \pm$ $0.5{ }^{\circ} \mathrm{C}$ for $20-24 \mathrm{~h}$. From these cultures, the organisms were directly suspended in $5 \mathrm{~mL}$ of sterile saline solution $(\mathrm{NaCl} 0.9 \% \mathrm{w} / \mathrm{v})$. The turbidity of each suspension was adjusted to 0.5 McFarland standard using a spectrophotometer (Milton-Roy Spectronic 601, USA) at $625 \mathrm{~nm}$, corresponding to $\mathrm{ca}$. $1 \times 10^{8}$ colony-forming units (CFU) $/ \mathrm{mL}$. The suspension was further diluted $1: 10$ with sterile saline solution. A $2-\mu \mathrm{L}$ aliquot of each suspension was applied to MHA plates containing increasing amounts of the drug so as to obtain ca. $10^{4} \mathrm{CFU} / \mathrm{spot}$. The plates were incubated at $35 \pm 0.5{ }^{\circ} \mathrm{C}$ and examined for the appearance of growth after $24 \mathrm{~h}$, extended up to $72 \mathrm{~h}$ where necessary. MIC was recorded as the lowest concentration of antimicrobial agent that completely inhibited growth, disregarding a single colony or a faint haze caused by the inoculum.

\section{Determination of synergy between the antibiotics and the phenothiazines}

Presence of synergy and its degree was confirmed by Checkerboard method with microtiter trays using cation-adjusted MuellerHinton broth (CAMHB) as described elsewhere [5]. Oxacillin was tested at concentrations of 1 to $512 \mu \mathrm{g} / \mathrm{mL}$, and phenothiazines at 0.625 to $512 \mu \mathrm{g} / \mathrm{mL}$. The trays were prepared and kept for a maximum of one week at $-20{ }^{\circ} \mathrm{C}$ before use. Oxacillin was dispensed alone in the first row, and phenothiazine was dispensed in the first column. To each well, $6 \mu \mathrm{L}$ of an inoculum of ca. $10^{7} \mathrm{CFU} / \mathrm{mL}$ was added to make a final concentration of ca. $10^{5} \mathrm{CFU} / \mathrm{mL}$. The trays were incubated aerobically at $35 \pm 0.5{ }^{\circ} \mathrm{C}$ for $24 \mathrm{~h}$. For each run, ATCC reference strains served as control. Cultures that contained neither a phenothiazine nor antibiotic served as growth control. MIC was read as the lowest concentration of antimicrobial agents showing no visible growth or only a faint haze. MICs were determined for each agent individually and in combination.

\section{Data analysis}

All determinations were repeated three times. For clinical isolates, $\mathrm{MIC}_{50}$ was determined, which is the minimum concentration inhibiting $50 \%$ of isolates. Synergy was determined by calculating the fractional inhibitory concentration (FIC) index as in Eq 1.

$F I C$ index $=F I C_{A}+F I C_{B}=[A] / M I C_{A}+[B] / M I C_{B} \ldots \ldots$ (1)

where $[A]$ is the inhibitory concentration of the organism to drug $A$ when combined with $B$, $\mathrm{MIC}_{A}$ is the MIC of the organism to drug $A$ alone, $\mathrm{FIC}_{\mathrm{A}}$ is the $\mathrm{FIC}$ of $\operatorname{drug} \mathrm{A}$, and $[\mathrm{B}]$, $\mathrm{MIC}_{B}$, and $\mathrm{FIC}_{B}$ are defined in the same fashion for drug $B$. FIC index was interpreted as follows: $<0.5$, synergy; $0.50-0.75$, partial synergy; 0.76 - 1.0, additive effect; > 1.0 4.0, indifference; and > 4.0, antagonism [6]. For clinical isolates, median FICs were calculated using SPSS 17.0 software (SPSS Inc., USA).

\section{RESULTS}

The MICs of test strains to oxacillin and phenothiazines are presented in Table 1. Oxacillin inhibited MSSA ATCC 29213 and MRSA ATCC 33591 at $2 \mu \mathrm{g} / \mathrm{mL}$ and 256 $\mu \mathrm{g} / \mathrm{mL}$, respectively, which are within the reference range for these control strains. This antibiotic also inhibited all clinical isolates of MRSA at $256 \mu \mathrm{g} / \mathrm{mL}$. All the phenothiazines inhibited the growth of MSSA and MRSA strains, with thioridazine being the most potent inhibitory agent. MRSA isolates were either highly sensitive $(<50 \mu \mathrm{g} / \mathrm{mL})$ or moderately sensitive $(50-400 \mu \mathrm{g} / \mathrm{mL})$ to the phenothiazines. The phenothiazines inhibited $S$. aureus strains regardless of their susceptibility status to oxacillin.

The degree of synergy between oxacillin and the phenothiazines against the MRSA isolates, determined by Checkerboard techni- 
Table 1: Susceptibility of Staphylococcus aureus strains determined by the agar dilution method

\begin{tabular}{|c|c|c|c|c|c|c|c|c|c|c|c|c|c|c|c|}
\hline \multirow[t]{3}{*}{ Strain } & \multicolumn{15}{|c|}{ Minimum Inhibitory Concentration (MIC) } \\
\hline & \multirow{2}{*}{$\frac{\text { OXA }}{\mu \mathrm{g} / \mathrm{mL}}$} & \multicolumn{2}{|c|}{ CPZ } & \multicolumn{2}{|c|}{ TFP } & \multicolumn{2}{|c|}{ THZ } & \multicolumn{2}{|c|}{ TEP } & \multicolumn{2}{|c|}{ PFZ } & \multicolumn{2}{|c|}{$\mathbf{F Z}$} & \multicolumn{2}{|c|}{ PMZ } \\
\hline & & $\mu \mathrm{g} / \mathrm{mL}$ & $\mu \mathrm{M}$ & $\mu \mathrm{g} / \mathrm{mL}$ & $\mu \mathrm{M}$ & $\mu \mathrm{g} / \mathrm{mL}$ & $\mu \mathrm{M}$ & $\mu \mathrm{g} / \mathrm{mL}$ & $\mu \mathrm{M}$ & $\mu \mathrm{g} / \mathrm{mL}$ & $\mu \mathrm{M}$ & $\mu \mathrm{g} / \mathrm{mL}$ & $\mu \mathrm{M}$ & $\mu \mathrm{g} / \mathrm{mL}$ & $\mu \mathrm{M}$ \\
\hline MSSA & $2^{*}$ & 16 & 45 & 16 & 33.3 & 16 & 39.3 & 16 & 25.3 & 256 & 633.7 & 16 & 31.3 & 128 & 398.9 \\
\hline MRSA & 256 & 16 & 45 & 32 & 66.6 & 16 & 39.3 & 16 & 25.3 & 256 & 633.7 & 32 & 62.7 & 128 & 398.9 \\
\hline$M_{1}$ & 256 & 16 & 45 & 16 & 33.3 & 8 & 19.7 & 16 & 25.3 & 256 & 633.7 & 16 & 31.3 & 128 & 398.9 \\
\hline $\mathrm{M}_{2}$ & 256 & 16 & 45 & 32 & 66.6 & 8 & 19.7 & 16 & 25.3 & 256 & 633.7 & 16 & 31.3 & 128 & 398.9 \\
\hline$M_{3}$ & 256 & 16 & 45 & 32 & 66.6 & 8 & 19.7 & 16 & 25.3 & 256 & 633.7 & 16 & 31.3 & 128 & 398.9 \\
\hline $\mathrm{M}_{4}$ & 256 & 16 & 45 & 32 & 66.6 & 8 & 19.7 & 16 & 25.3 & 256 & 633.7 & 16 & 31.3 & 128 & 398.9 \\
\hline$M_{5}$ & 256 & 16 & 45 & 32 & 66.6 & 8 & 19.7 & 16 & 25.3 & 256 & 633.7 & 16 & 31.3 & 128 & 398.9 \\
\hline$M_{6}$ & 256 & 16 & 45 & 32 & 66.6 & 8 & 19.7 & 16 & 25.3 & 256 & 633.7 & 16 & 31.3 & 128 & 398.9 \\
\hline$M_{7}$ & 256 & 16 & 45 & 32 & 66.6 & 8 & 19.7 & 16 & 25.3 & 256 & 633.7 & 16 & 31.3 & 128 & 398.9 \\
\hline$M_{8}$ & 256 & 16 & 45 & 32 & 66.6 & 8 & 19.7 & 16 & 25.3 & 256 & 633.7 & 16 & 31.3 & 128 & 398.9 \\
\hline$M_{9}$ & 256 & 16 & 45 & 16 & 33.3 & 8 & 19.7 & 16 & 25.3 & 256 & 633.7 & 16 & 31.3 & 128 & 398.9 \\
\hline $\mathrm{M}_{10}$ & 256 & 16 & 45 & 32 & 66.6 & 8 & 19.7 & 16 & 25.3 & 256 & 633.7 & 16 & 31.3 & 128 & 398.9 \\
\hline $\mathrm{MIC}_{50}$ & 256 & 16 & 45 & 32 & 66.6 & 8 & 19.7 & 16 & 25.3 & 256 & 633.7 & 16 & 31.3 & 128 & 398.9 \\
\hline
\end{tabular}

OXA: Oxacillin, CPZ: chlorpromazine, TFP: trifluoperazine, THZ: thioridazine, TEP: thiethyl perazine, PFZ: perphenazine, FZ: fluphenazine, PMZ:

promethazine. MSSA: S. aureus ATCC 29213, MRSA: Methicillin-resistant S. aureus ATCC 33591. M1-M10: 10 clinical MRSA isolates used in the study. ${ }^{*}$ Experiments were repeated three times and the results were the same. MIC 50 represents the concentration which is able to inhibit $50 \%$ of clinical MRSA isolates. 
que, are shown in Table 2. Various combinations of oxacillin with phenothiazines inhibited most of the MRSA strains at concentrations well below their minimum inhibitory concentrations. This inhibitory effect was significantly expressed (synergistic or partially synergistic). The highest number of synergistic combinations (FIC < 0.5) were seen with chlorpromazine and perphenazine. Surprisingly, perphenazine remarkably potentiated the effect of oxacillin against MRSA isolates in spite of its rather high MIC when used alone (256 $\mu \mathrm{g} / \mathrm{mL})$. However, thioridazine also reversed antibiotic resistance at a concentration as low as 4 $\mu \mathrm{g} / \mathrm{mL}$. In contrast, no significant synergy was observed for combinations of oxacillin and promethazine. In some cases, phenolthiazines reduced the MIC of oxacillin to a clinically significant level, i.e., $2 \mu \mathrm{g} / \mathrm{mL}$.

\section{DISCUSSION}

The antimicrobial activity of phenothiazines has been known since the time of Ehrlich (1891) [7]; nevertheless, enhancement of the activity of conventional antibiotics by these and other compounds has only recently been a subject of interest. To the best of our knowledge, phenothiazines such as thiethylperazine have not previously been evaluated for their antibiotic resistancemodifying activity.

This investigation was carried out in two steps. First, the MIC of the drugs was determined by agar dilution method. The MICs obtained for MSSA ATCC 29213 and MRSA isolates were $2 \mu \mathrm{g} / \mathrm{mL}$ and $256 \mu \mathrm{g} / \mathrm{mL}$, respectively, confirming both the accuracy of the method and resistance of the clinical isolates based on CLSI guidelines [4]. On the other hand, the results implied high $(\mathrm{MIC}<$ $50 \mu \mathrm{g} / \mathrm{mL}$ ) to moderate $(50-400 \mu \mathrm{g} / \mathrm{mL}$ ) activity of phenothiazines against the MRSA isolates [6]. The MICs obtained for chlorpromazine, trifluoperazine, thioridazine and promethazine are consistent with those reported previously [8-10]. The antibacterial potency of the drugs was not linked to their other pharmacologic activities, and this is in agreement with results of earlier investigations [11]. To understand the stoichiometric relationship between the phenothiazines and their antibacterial activity, their potency was calculated on the basis of their molar concentration. Yet, their antibacterial activity in micromolar $(\mu \mathrm{M})$ concentrations was comparable to that in $\mu \mathrm{g} / \mathrm{mL}$. Furthermore, the results show that phenothiazines have an antimicrobial effect against $S$. aureus strains regardless of their resistance pattern to oxacillin. A similar finding was also reported for $S$. aureus using the combination of chlorpromazine and thioridazine [12]. This may be attributed to of the fact that these non-antibiotics and oxacillin exert their activities at different sites. Phenothiazines inhibit enzyme systems which are dependent upon $\mathrm{Ca}^{2+}$, such as those involved in generating cellular energy from hydrolysis of ATP, by preventing the transport of calcium $\left(\mathrm{Ca}^{2+}\right)$ through $\mathrm{Ca}^{2+}$ binding proteins such as calmodulin [13]. Among these phenothiazine-sensitive systems are transporters that extrude from the cell a variety of harmful agents such as antibiotics and hence protect the cell from these agents [14].

Steady state plasma concentrations of chlorpromazine, fluphenazine, promethazine, thioridazine, trifluoperazine and perphenazine have already been found to be $0.002-0.12$, $0.002-0.004,0.002-0.018,0.05-0.5,0.001$ - 0.004 and $0.0003-0.025 \mu \mathrm{g} / \mathrm{mL}$, respectively [15]. Our in vitro findings revealed that phenothiazines inhibited MRSA isolates at concentrations which are well beyond their plasma concentrations. However, phenothiazines such as thioridazine are concentrated as much as 100 -fold by macrophages [16]. Killing of phagocytosed $S$. aureus, an intracellular infection of the human macrophage, by nonkilling macrophages is enhanced by concentrations of thioridazine that are well below those employed for the therapy of psychoses [16]. Additionally, in vivo experiments with Swiss mice have 
Hadji-nejad et al

Table 2: Synergy between oxacillin and phenothiazines against methicillin-resistant $S$. aureus (Checkerboard method)

\begin{tabular}{|c|c|c|c|c|c|c|c|c|c|c|c|c|c|c|}
\hline \multirow{2}{*}{$\begin{array}{l}\text { Strain } \\
M_{1}\end{array}$} & \multicolumn{2}{|c|}{$O X A+C P Z$} & \multicolumn{2}{|c|}{ OXA + TFP } & \multicolumn{2}{|c|}{ OXA + THZ } & \multicolumn{2}{|c|}{ OXA + TEP } & \multicolumn{2}{|c|}{ OXA + PFZ } & \multicolumn{2}{|c|}{$O X A+F Z$} & \multicolumn{2}{|c|}{$O X A+P M Z$} \\
\hline & $4 / 256^{*}$ & $8 / 32$ & $8 / 256$ & $16 / 32$ & $32 / 256$ & $4 / 8$ & $16 / 256$ & $8 / 16$ & $2 / 256$ & $64 / 256$ & $2 / 256$ & $8 / 16$ & $4 / 256$ & $64 / 128$ \\
\hline $\mathrm{M}_{2}$ & $2 / 256$ & $16 / 32$ & $32 / 256$ & $16 / 32$ & $16 / 256$ & $8 / 16$ & $16 / 256$ & $8 / 16$ & $2 / 256$ & $32 / 128$ & $4 / 256$ & $8 / 32$ & $4 / 256$ & $64 / 128$ \\
\hline$M_{3}$ & $2 / 256$ & $8 / 32$ & $8 / 256$ & $8 / 16$ & $8 / 256$ & $4 / 8$ & $8 / 256$ & $2 / 16$ & $2 / 256$ & $64 / 256$ & $2 / 256$ & $8 / 16$ & $8 / 256$ & $64 / 128$ \\
\hline $\mathrm{M}_{4}$ & $4 / 256$ & $8 / 32$ & $16 / 256$ & $8 / 32$ & $4 / 256$ & $8 / 16$ & $4 / 256$ & $8 / 16$ & $2 / 256$ & $64 / 256$ & $8 / 256$ & $16 / 32$ & $64 / 256$ & $32 / 64$ \\
\hline$M_{5}$ & $2 / 256$ & $16 / 32$ & $8 / 256$ & $16 / 32$ & $2 / 256$ & $8 / 16$ & $16 / 256$ & $8 / 16$ & $16 / 256$ & $64 / 128$ & $4 / 256$ & $8 / 16$ & $16 / 256$ & $64 / 128$ \\
\hline$M_{6}$ & $2 / 256$ & $8 / 32$ & $2 / 256$ & $8 / 32$ & $2 / 256$ & $4 / 8$ & $16 / 256$ & $4 / 16$ & $4 / 256$ & $128 / 256$ & $8 / 256$ & $4 / 16$ & $16 / 256$ & $32 / 64$ \\
\hline$M_{7}$ & $8 / 256$ & $8 / 32$ & $16 / 256$ & $16 / 32$ & $2 / 256$ & $4 / 16$ & $16 / 256$ & $4 / 16$ & $2 / 256$ & $64 / 256$ & $4 / 256$ & $16 / 32$ & $32 / 256$ & $32 / 64$ \\
\hline$M_{8}$ & $16 / 256$ & $16 / 32$ & $2 / 256$ & $16 / 32$ & $4 / 256$ & $8 / 16$ & $16 / 256$ & $8 / 16$ & $2 / 256$ & $64 / 256$ & $16 / 256$ & $8 / 16$ & $16 / 256$ & $64 / 128$ \\
\hline$M_{9}$ & $2 / 256$ & $16 / 32$ & $4 / 256$ & $8 / 16$ & $2 / 256$ & $8 / 16$ & $8 / 256$ & $8 / 16$ & $2 / 256$ & $64 / 128$ & $16 / 256$ & $8 / 16$ & $2 / 256$ & $64 / 128$ \\
\hline$M_{10}$ & $2 / 256$ & $16 / 32$ & $8 / 256$ & $8 / 16$ & $2 / 256$ & $4 / 16$ & $4 / 256$ & $8 / 16$ & $4 / 256$ & $128 / 256$ & $32 / 256$ & $8 / 16$ & $2 / 256$ & $64 / 128$ \\
\hline $\begin{array}{l}\text { Median } \\
\text { FIC }\end{array}$ & \multicolumn{2}{|c|}{0.4} & \multicolumn{2}{|c|}{0.53} & \multicolumn{2}{|c|}{0.52} & \multicolumn{2}{|c|}{0.53} & \multicolumn{2}{|c|}{0.26} & \multicolumn{2}{|c|}{0.52} & \multicolumn{2}{|c|}{0.55} \\
\hline MRSA & $2 / 256$ & $16 / 32$ & $2 / 256$ & $16 / 32$ & $2 / 256$ & $8 / 16$ & $8 / 256$ & $8 / 16$ & $2 / 256$ & $128 / 256$ & $4 / 256$ & $8 / 32$ & $4 / 256$ & $64 / 128$ \\
\hline
\end{tabular}

OXA: Oxacillin, CPZ: chlorpromazine, TFP: trifluoperazine, THZ: thioridazine, TEP: thiethyl perazine, PFZ: perphenazine, FZ: fluphenazine, PMZ: promethazine. MRSA: Methicillin-resistant $\mathrm{S}$. aureus ATCC 33591, $\mathrm{M}_{1}-\mathrm{M}_{10}$ : 10 clinical MRSA isolates used in the study.

* Data are presented as drug inhibitory concentration in combination/MIC of drug alone (both in $\mu \mathrm{g} / \mathrm{mL}$ ). Each experiment was repeated three times and the results

were the same. Cells with greybackground represent synergy between oxacillin and the respective phenothiazine. Other cells denote partial synergy.

Fractional Inhibitory Concentration (FIC) index interpretation: <0.5, synergy; $0.5-0.75$, partial synergy; $0.76-1.0$, additive effect; $>1.0-4.0$, indifference; > 4.0, antagonism. 
demonstrated the potential antibacterial activity of these compounds [9].

In the next step, synergy between oxacillin and phenothiazines were investigated by Checkerboard technique. The results showed that susceptibility of MRSA strains to oxacillin was significantly affected by the phenothiazines, particularly chlorpromazine and perphenazine. The reduction of oxacillin resistance from 256 to $2 \mu \mathrm{g} / \mathrm{mL}$ was identical for these two pheothiazines. These results are comparable to those of other researchers $[2,16]$, who found a reduction in MIC of oxacillin against MRSA when phenothiazines derivatives were added to the medium. The phenothiazines reduced oxacillin resistance to $1 / 2$ or $1 / 4$ of their MICs a phenomenon that was observed in a previous report [2]. Furthermore, thioridazine showed the most potent activity among phenothiazines, and modified oxacillin resistance at concentrations lower than those of other phenothiazines. Thioridazine, though has less severe side effects than chlorpromazine, has been shown to have cardiotoxic side effects and, as a result, was withdrawn in 2005. Still, thioridazine is of therapeutic interest since the (S)-enantiomer, (S)thioridazine, is less toxic [17] and a more effective potentiator of antibiotics than the other thioridazine derivative, (R)-thioridazine [8].

Although this study did not address the mechanism(s) by which phenothiazines mitigate the resistance of MRSA strains to oxacillin, resistance of staphylococci to oxacillin is mediated by mecA, an 'acquired' gene that encodes a variant (PBP2a) of penicillin-binding protein (PBP2) and which has low affinity for all beta-lactams. Transposon inactivation of the structural gene of the $S$. aureus PBP2 of a highly methicillinresistant strain resulted in a reduction of methicillin resistance from 800 to $12 \mu \mathrm{g} / \mathrm{mL}$ though normal amounts of PBP2a were produced by this transposon mutant [18]. This massive reduction of methicillin resistance was recently shown to involve the deactivation of the transglycolase domain of PBP2 and not its transpeptidase domain [19]. It is tempting to suggest that the similar reduction of oxacillin resistance from 256 to 2 $\mu \mathrm{g} / \mathrm{mL}$ found in our work may be mostly due to such deactivation by phenothiazines such as chlorpromazine, in that this compound is well known to deactivate a large number of enzymes [20]. Moreover, the addition of the combinations of neurotropic drugs and $\beta$ lactam oxacillin led to lower levels of specific RNAs and proteins, indicating their interference with DNA-based processes [21]. Thioridazine also induces ultra-structural changes in MRSA such as alteration of the structure of the cell envelope, resulting in bacterial lysis at clinically relevant concentrations [3].

\section{CONCLUSION}

Phenothiazines, including thiethylperazine, can significantly reduce the resistance of MRSA strains to oxacillin in vitro. However, further investigations are necessary to elaborate the mechanism through which phenothiazines exert this resistancemodifying effect. Finally, the question of whether the concentrations of phenothiazines that are effective in vitro against phagocytosed staphylococci are rendered less effective in vivo by physiologically limiting parameters such as proteins binding of the drugs or their conversion to inactive metabolites produced by the liver, must be answered, and therefore, should be a subject of further study before consideration of the compound for clinical trial. Although synergy was observed at concentrations higher than those that phenothiazines reach to in vivo, the potential offered by non-antibiotics corroborate further animal experiments as well as clinical trials to establish their clinical relevance.

\section{ACKNOWLEDGMENT}

This study was supported by Grant no. 1048 from Shahid Beheshti University of Medical Sciences. We express our best gratitude to 
Tehran Chemie, Chemidarou, Pars Minoo and Darou Pakhsh pharmaceutical companies for providing us with phenothiazines.

\section{REFERENCES}

1. Kristiansen JE. Antimicrobial activity of nonantibiotics. Acta Path Microbiol Scand 1992; 100(Suppl.): 7-19.

2. Kristiansen MM, Leandro $C$, Ordway $D$, Martins $M$, Viveiros M, Pacheco T, Kristiansen JE, Amaral $L$. Phenothiazines alter resistance of methicillin-resistant strains of Staphylococcus aureus (MRSA) to oxacillin in vitro. Int $J$ Antimicrob Agents 2003; 22: 250-253.

3. Martins $M$, Bleiss $W$, Marko $A$, Ordway $D$, Viveiros $M$, Leandro $C$, Pacheco $T$, Molnár J, Kristiansen JE, Amaral L. Clinical concentrations of thioridazine enhance the killing of intracellular methicillin-resistant Staphylococcus aureus: an in vivo, ex vivo and electron microscopy study. In Vivo 2004; 18: 787-794.

4. Clinical and Laboratory Standards Institute (CLSI). Performance standards for antimicrobial susceptibility testing. Sixteenth informational supplement. Document M100-S16. Wayne, PA: CLSI, 2006.

5. Pillai SK.; Moellering RC. Eliopoulos George M. Antimicrobial combinations. In: Lorian Victor, editor. Antibiotics in laboratory medicine. 5th ed. Baltimore: Wiliams \& Wilkins; 2005. p 365441.

6. Basu LR, Mazumdar K, Dutta NK, Karak P, Dastidar SG. Antibacterial property of the antipyschotic agent prochlorperazine, and its synergism with methdilazine. Microbiol Res 2005; 160: 95100.

7. Guttmann $P$, Ehrlich $P$. Über die Wirkung des Methylenblau bei Malaria. Berliner Klinische Wochenschrift 1891; 39: 953-956.

8. Kristiansen JE, Hendricks O, Delvin T, Butterworth TS, Aagaard L, Christensen JB, Flores VC, Keyzer K. Reversal of resistance in microorganisms by help of non-antibiotics. J Antimicrob Chemother 2007; 59: 1271-1279.

9. Mazumder $R$, Chaudhuri SR, Mazumdar A. Antimicrobial potentiality of a phenothiazine group of antipyschotic drug-prochlorperazine. Indian J Exp Biol 2002; 40: 828-830.

10. Hendricks $O$, Butterworth TS, Kristiansen JE. The in-vitro antimicrobial effect of non-antibiotics and putative inhibitors of efflux pumps on Pseudomonas aeruginosa and
Staphylococcus aureus. Int $J$ Antimicrob Agents 2003; 22: 262-264.

11. Kristiansen JE, Mortensen I. Antibacterial effect of four phenothiazines. Pharmacol Toxicol 1987; 60: 100-103.

12. Kaatz, GW, Mougdal VV, Seo SM, Kristiansen JE. Phenothiazines and thioxanthenes inhibit multidrug efflux pump activity in Staphylococcus aureus. Antimicrob Agents Chemother 2003, 47: 719-726.

13. Garcia JJ, Tuena de Gomez-Puyou M, GomezPuyou A. Inhibition by trifluoperazine of ATP synthesis and hydrolysis by particulate and soluble mitochondrial F1: competition with H2PO4. J Bioenerg Biomembr 1995; 27: 127136.

14. Piddock LJV. Clinically relevant chromosomally encoded multidrug resistance efflux pumps in bacteria. Clin Microbiol Rev 2006; 19: 382 402.

15. Moffart, Anthony C., Ed., Clarke's isolation and identification of drugs. 2nd ed. London: The Pharmaceutical Press; 1986.

16. Ordway $D$, Viveiros $M$, Leandro $C$, Arroz $M J$, Amaral L. Intracellular activity of clinical concentrations of phenothiazines including thioridazine against phagocytosed Staphylococcus aureus. Int J Antimicrob Agents 2002; 20: 34-43.

17. Svendsen $C N$, Froimowitz $M$, Hrbek $C$, Campbell $A$, Kula N, Baldessarini RJ, Cohen BM, Babb S, Teicher MH, Bird ED. Receptor affinity, neurochemistry and behavioral characteristics of the enantiomers of thioridazine: evidence for different stereoselectivities at D1 and D2 receptors in rat brain. Neuropharmacology 1988; 27: 1117-1124.

18. Pinho MG, Ludovice AM, Wu S, De Lencastre $H$. Massive reduction in methicillin resistance by transposon inactivation of the normal PBP2 in a methicillin-resistant strain of Staphylococcus aureus. Microb Drug Resist 1997; 3: 409-413.

19. Pinho MG, de Lencastre H, Tomasz A. An acquired and a native penicillin-binding protein cooperate in building the cell wall of drugresistant staphylococci. Proc Natl Acad Sci USA 2001; 98: 10886-10891.

20. Amaral $L$, Viveiros $M$, Kristiansen JE. Phenothiazines: potential alternatives for the management of antibiotic resistant infections of tuberculosis and malaria in developing countries. Trop Med Int Health 2001; 6: 10161022.

21. Klitgaard JK, Skov MN, Kallipolitis BH, Kolmos HJ. Reversal of methicillin resistance in Staphylococcus aureus by thioridazine. J Antimicrob Chemother 2008; 62: 1215-1221. 